\title{
Potentiation of Fas-mediated apoptosis by an engineered glycosylphosphatidylinositol-linked Fas
}

\author{
P Legembre ${ }^{1}, \mathrm{P}$ Moreau $^{2}, \mathrm{~S}$ Daburon ${ }^{1}$, \\ J-F Moreau ${ }^{1}$ and J-L Taupin ${ }^{\star, 1}$ \\ 1 Laboratoire d'Immunologie, CNRS UMR 5540, Université de Bordeaux 2 , \\ bâtiment $1 \mathrm{~b}, 146$ rue Léo-Saignat, 33076 Bordeaux, France \\ 2 Laboratoire de Biogenèse Membranaire, CNRS UMR 5544, Université de \\ Bordeaux 2, 146 rue Léo-Saignat, 33076 Bordeaux, France \\ * Corresponding author: J-L Taupin, Tel: +33-5-57-57-14-71; \\ Fax: +33-5-57-57-14-72; E-mail: jean-luc.taupin@umr5540.u-bordeaux2.fr
}

Received 27.3.01; revised 11.9.01; accepted 20.9.01

Edited by J Tschopp

\begin{abstract}
FasL and TRAIL are apoptotic ligands of the TNF-like cytokines family, acting via activation of the transmembrane death domain containing receptors Fas for FasL, and DR4 or DR5 for TRAIL. A glycosylphosphatidylinositol-linked TRAIL receptor called DcR1 behaves as a decoy receptor inhibiting TRAIL-mediated cell death in several cellular systems. We engineered and stably expressed a chimeric GPI-linked Fas receptor (Fas-GPI) in T-lymphocyte cell lines constitutively expressing functional transmembrane Fas. Surprisingly, despite lacking the death domain region of functional Fas, Fas-GPI was able to significantly increase Fas-mediated cell death triggered by membrane bound or soluble FasL, whereas engagement of Fas-GPI alone did not trigger apoptosis. This potentiating effect, but not transmembrane Fas activation, was selectively inhibited by protein kinase $C$ activation with phorbol esters, demonstrating that Fas-GPI activated a specific synergistic signal transduction pathway. Fas-GPI and transmembrane Fas were localized in distinct membrane compartments, since Fas-GPI, but not transmembrane Fas, was found in the glycolipid-rich membrane microdomains. These results suggest that apoptosis induced by members of this ligand/receptors family may be differentially modulated through other and parallel signalling pathways.

Cell Death and Differentiation (2002) 9, 329-339. DOI: 10.1038/ sj/cdd/4400960
\end{abstract}

Keywords: GPI; Fas; apoptosis; microdomains

Abbreviations: TNF: Tumor necrosis factor; GPI: glycosylphosphatidylinositol; mAb: monoclonal antibody; PIPLC: phosphatidylinositol-specific phospholipase C; PMA: phorbol 12-myristate 13acetate

\section{Introduction}

The Fas ligand (FasL) receptor Fas and the TRAIL receptors are apoptosis inducers which belong to the Tumor Necrosis
Factor (TNF) receptor superfamily. Fas-mediated apoptosis is an important regulator of the immune response as Fas activation has been involved in the negative regulation of the activated lymphocytes via the activation-induced cell death (AICD), ${ }^{1-3}$ and in the deletion of antigen-presenting cells following the contact with the activated T-lymphocytes. ${ }^{4-6}$ Inhibition of Fas-mediated cell death would therefore have for consequences to increase the lifetime of activated Tlymphocytes or of antigen-presenting cells, which may lead to an increased efficiency of the immune response.

Among the TRAIL receptors (for review, $\mathrm{see}^{7}$ ) is one called DCR1, LIT, TRAIL-R3 or TRID ${ }^{8-12}$ which is endowed with an ability to bind TRAIL comparable to that of the functional receptors called DR4 and DR5, ${ }^{13,14}$ but is glycosylphosphatidylinositol-linked (GPI-linked) to the membrane and therefore incapable of transducing any death signal. Several reports showed that DcR1 inhibits TRAILmediated cell death when overexpressed in various TRAILsensitive cell lines, $, 8,12,15$ and that PIPLC treatment of DcR1 expressing cells cleaves the GPI-linked protein off the membrane and increases susceptibility to TRAIL. ${ }^{12,16}$ Based on the findings described for the TRAIL receptor DcR1, we engineered a chimeric GPI-linked Fas receptor, which we called Fas-GPI. Fas-GPI was stably transfected into human T-lymphoblastoid cell lines, which constitutively express functional transmembrane Fas. In these transfectants, we analyzed whether the co-crosslinking GPI-linked Fas together with transmembrane Fas receptors via FasL would interfere in the apoptotic signal normally delivered via Fas.

\section{Results \\ Obtention and characterization of Jurkat transfectants expressing Fas-GPI}

The cDNA sequence encoding the transmembrane and intracellular regions of the FAS antigen was deleted and exchanged with the sequence encoding the 37 carboxyterminal amino acids of DAF (Decay Accelerating Factor, or CD55), which is responsible for the glycosylphosphatidylinositol (GPI) membrane targeting of DAF. ${ }^{17-21}$ At the junction between the Fas sequence and the GPI-anchoring region, we intercalated the c-myc sequence recognized by the $9 \mathrm{E} 10$ monoclonal antibody (mAb). The FasmycDAF chimeric construct, herein called Fas-GPI, was transfected in the human T-lymphoblastoid Jurkat cell line and stable transfectants were isolated by limiting dilution. The Jurkat cell line constitutively expressed transmembrane full length Fas, and was sensitive to the apoptosis induced by FasL. Although it was anticipated that the expression of membrane-bound Fas-GPI would increase the total amount of Fas on the cell surface as analyzed by flow cytometry, a staining with the 
anti-c-myc antibody 9E10 was used to discriminate between the transmembrane and GPI-linked forms. Three representative transfectants expressing various amounts of Fas-GPI are shown in Figure 1. They were labelled with the anti-cmyc mAb, and displayed low, intermediate and high levels of Fas-GPI, while the mock-transfected Jurkat cell line was not labelled with this antibody (Figure 1A). The transfectants were also stained with an anti-Fas mAb (clone 5D7, IgG1). They all displayed an increased expression of Fas when compared to the mock-transfected cell line, which paralleled that noted with the anti-c-myc staining (Figure 1C). The same staining pattern was also obtained with three other anti-Fas IgG1 mAbs directed against different epitopes on Fas (BG27, B-G30 and DX2), as well as with the anti-Fas agonistic IgM 7C11 (data not shown), which ruled out a putative variability in epitope accessibility for the mAbs on different transfectants. When the transfectants and the mocktransfected Jurkat were treated with phosphatidylinositolspecific phospholipase C (PIPLC), which specifically cleaves the protein in the GPI anchor, the c-myc epitope staining almost completely disappeared (Figure 1B), and the intensity of the Fas staining on the three Fas-GPI transfectants decreased to the basal level obtained with the mocktransfected Jurkat (Figure 1D). These results demonstrated that the Fas-GPI protein was effectively GPI-linked and that the expression of the chimeric Fas did not alter the membrane expression level of the endogenous wild-type transmembrane Fas, which remained unchanged. Therefore, any further change in the sensitivity of the Jurkat Fas-GPI transfectants towards FasL should not be attributable to a change in the expression level of endogenous Fas.

\section{Expression of Fas-GPI enhanced susceptibility to cell death induced by membrane-bound and soluble FasL}

We next assessed the functionality of the Fas system in these transfectants, and examined whether the expression of FasGPI could alter the sensitivity of the Jurkat cells to the apoptosis induced by FasL (Figure 2). The mock-transfected Jurkat cell line as well as the transfectants expressing low, intermediate and high levels of Fas-GPI were labelled with ${ }^{51} \mathrm{Cr}$, and incubated with the $1 \mathrm{~A} 12$ murine effector cells, which stably expressed functional membrane human FasL, or with its untransfected counterpart WR19L, at various effector/ target ratios. As expected, the mock-transfected Jurkat cell line was efficiently killed by the FasL-expressing $1 \mathrm{~A} 12$ cell line, but not by the WR19L FasL-negative control cell line. The Fas-GPI transfectants were also efficiently killed by the $1 \mathrm{~A} 12$ cell line but not by the WR19L (Figure 2C and A respectively). We noticed that these transfectants were more sensitive to the $1 \mathrm{~A} 12$ cell line than the mock-transfected cell line, and that their susceptibility to membrane-bound FasL paralleled the expression level of the Fas-GPI truncated receptor. In this 4-h assay, the maximum cell death doubled in the transfectant expressing the highest amount of Fas-GPI when compared to the mock-transfected cells, and the effector/target ratio required to reach $50 \%$ of the cell death plateau was diminished around eightfold.

To confirm the involvement of the Fas-GPI receptor in this increased sensitivity to apoptosis, the target Jurkat cells were pretreated with PIPLC in conditions which completely removed the chimeric Fas-GPI from the cell

\section{c-myc}

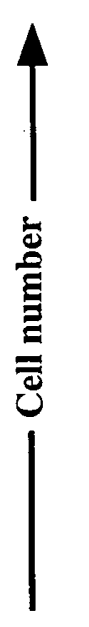

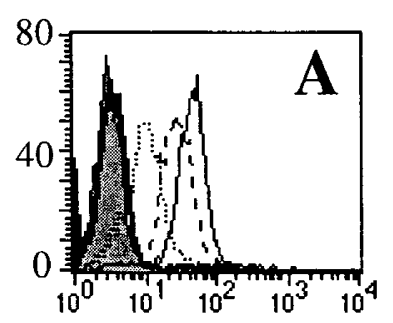

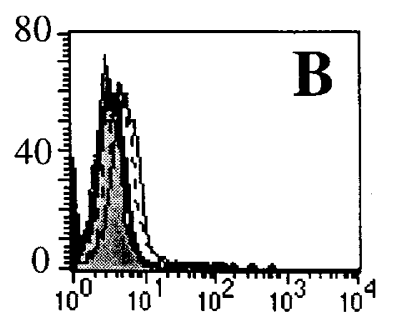

Fas
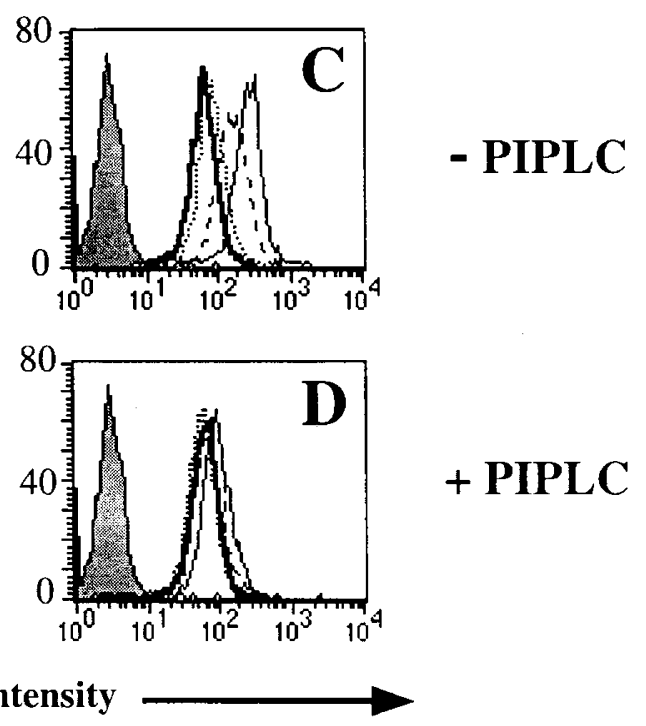

Figure 1 Membrane expression of Fas-GPI, and sensitivity of the GPI anchor to PIPLC. Surface staining for the c-myc epitope (A and B) and Fas extracellular domain ( $\mathbf{C}$ and $\mathbf{D}$ ) were analysed before ( $\mathbf{A}$ and $\mathbf{C}$ ) or after (B and $\mathbf{D})$ PIPLC treatment, for the mock-transfected Jurkat (thick line), and three representative Jurkat transfectants expressing low, intermediate or high amounts of Fas-GPI (dotted, dashed and thin solid line, respectively). The shaded histogram depicts the isotypematched negative control. This is one representative of four different experiments. These results were reproduced with three other transfectants displaying comparable levels of Fas-GPI 
- PIPLC

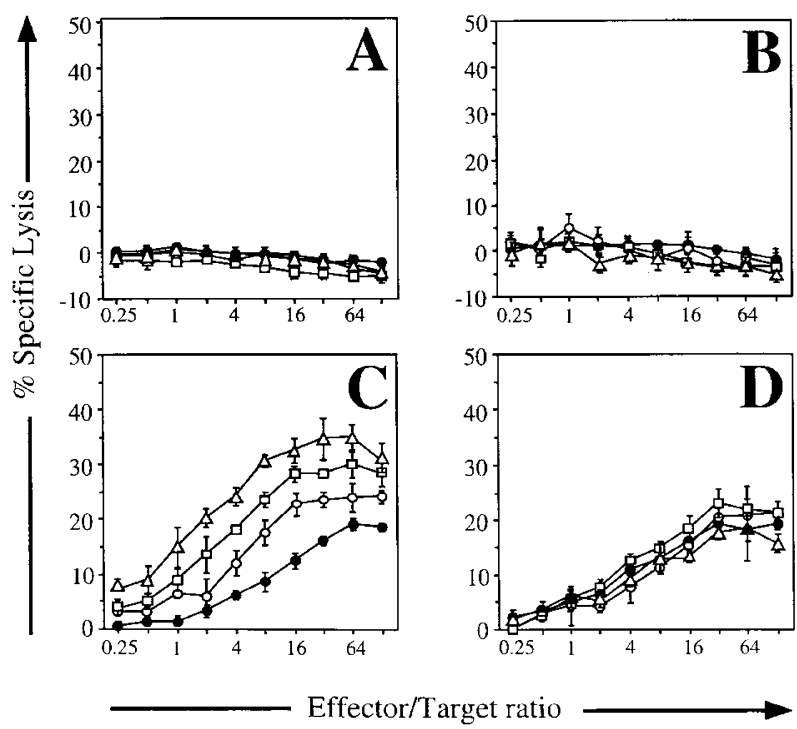

Figure 2 Fas-GPI enhanced Jurkat sensitivity to cytotoxicity mediated by membrane FasL and PIPLC restored basal sensitivity. Mock-transfected Jurkat cell line (filled circles) or the transfectants expressing low (open circles), intermediate (open squares) and high (open triangles) levels of FasGPI were treated (B and D) or not (A and $\mathbf{C}$ ) with PIPLC, labelled with ${ }^{51} \mathrm{Cr}$ and incubated with control WR19L (A and $\mathbf{B})$ or FasL-expressing $1 \mathrm{~A} 12$ cells ( $\mathbf{C}$ and D) for $4 \mathrm{~h}$ Cytotoxicity was assessed via measurement of ${ }^{51} \mathrm{Cr}$ release. Results are means of five experiments \pm S.D.

surface (see Figure 1), before the incubation with the effector WR19L or 1A12 cells (Figure 1B,D respectively). Treatment of the mock-transfected Jurkat cell line with PIPLC had no effect on its sensitivity to membrane-bound FasL. In contrast, the sensitivity to FasL of the PIPLCtreated transfectants expressing various amounts of FasGPI was drastically reduced, down to the level observed for the mock-transfected Jurkat cell line. Therefore, the gradual increase in sensitivity to FasL of the Fas-GPI transfectants was positively correlated to the increase in their membrane level of Fas-GPI.

We also used purified soluble recombinant FasL (sFasL), to confirm that the dose-dependent apoptosis enhancing effect we ascribed to Fas-GPI was not mediated by another GPI-linked membrane protein constitutively expressed by the Fas-GPI transfectants and mobilized by a specific ligand on the effector cell to enhance the death signal. For this purpose, the Fas-GPI transfectants and the mock-transfected Jurkat cell line were incubated with dilutions of sFasL, and sFasL-mediated cytotoxicity was measured after incubation, with the MTT viability assay carried out on a 20-h period of time. Figure 3 displays the results obtained for two independent transfectants expressing a high level of Fas-GPI. Both Fas-GPI transfectants displayed an increased sensitivity to sFasL, when compared with the control cell line (Figure $3 \mathrm{~A}$ ). When the cells were pretreated with PIPLC before sFasL addition, the sensitivity to sFasL of the mock-transfected Jurkat was not modified, while that of the Fas-GPI transfectants was decreased close to the level of the mock-transfected cells (Figure $3 \mathrm{~B}$ ). These results confirmed those obtained with membrane-bound FasL and clearly involved Fas-GPI in this enhancing effect while ruling out the putative role of other GPI-linked proteins.

\section{Fas-GPI did not trigger apoptosis on its own}

We analyzed the possibility that Fas-GPI could trigger cell death on its own following aggregation via FasL. We took advantage of the c-myc epitope to selectively cross-link FasGPI with the anti-c-myc 9E10 antibody, either directly plasticcoated or aggregated via a polyclonal anti-mouse IgG antiserum. No cytotoxic effect was seen over a wide range of concentrations of the $9 \mathrm{E} 10$ antibody (extending from $0.078 \mathrm{ng} / \mathrm{ml}$ to $40 \mu \mathrm{g} / \mathrm{ml}$ ), in the presence or in the absence of various concentrations of the anti-mouse $\operatorname{lgG}$ antiserum (from $0.156 \mathrm{ng} / \mathrm{ml}$ to $20 \mu \mathrm{g} / \mathrm{ml}$ ). Conversely, in similar conditions, the isotype-matched B-G27 anti-Fas antibody induced up to $20 \pm 3 \%(n=3)$ cell death in the transfectant expressing high levels of Fas-GPI (results not shown).

To confirm this result, we stably expressed Fas-GPI or full length Fas into the WR19L cell line, which does not express transmembrane Fas, and transfectants displaying high and comparable expression levels of Fas or Fas-GPI were obtained, as described above for the Jurkat cell line. We verified that Fas-GPI was lipid-linked on the cell surface, since PIPLC induced near complete disappearance of Fas-GPI (Figure 4C) but did not modify the expression of transmembrane Fas (Figure 4B). By using $1 \mathrm{~A} 12$ effector cells in a cell cytotoxicity assay, we showed that membrane FasL induced significant killing of WR19L cells expressing Fas whereas it had no effect at all on WR19L cells expressing Fas-GPI (Figure 4D). Therefore, Fas-GPI was not able to trigger cell death on its own, demonstrating that it is only capable of potentiating the agonistic effect mediated by the engagement of transmembrane Fas.

\section{Mobilization of Fas-GPI increased Fas-mediated DNA fragmentation and cleavage of caspases 3 and 8}

We next analyzed whether Fas-mediated cell death in the presence of Fas-GPI still occurred via apoptosis. Fas-GPI transfected or control Jurkat cells were mixed at a $1: 1$ ratio with WR19L or $1 \mathrm{~A} 12$ cells, in the presence or not of the antiFasL blocking mAb 10F2. After a $2 \mathrm{~h}$ incubation, the cell mixture was lysed and DNA fragmentation was analysed by agarose gel electrophoresis. As expected, DNA fragmentation (Figure 5A) occurred for mock-transfected Jurkat cells when incubated with $1 \mathrm{~A} 12$ cells, whereas it was not detectable in the presence of the control effector cells WR19L. Similar results were obtained for Fas-GPI Jurkat cells, but DNA fragmentation was clearly and reproducibly more pronounced than for mock-transfected Jurkat. For both target cell lines, FasL-mediated death was completely reversed with the FasL specific blocking mAb 10F2, indicating that cell death of the target cell lines was fully mediated by FasL. We next wondered whether the increase 


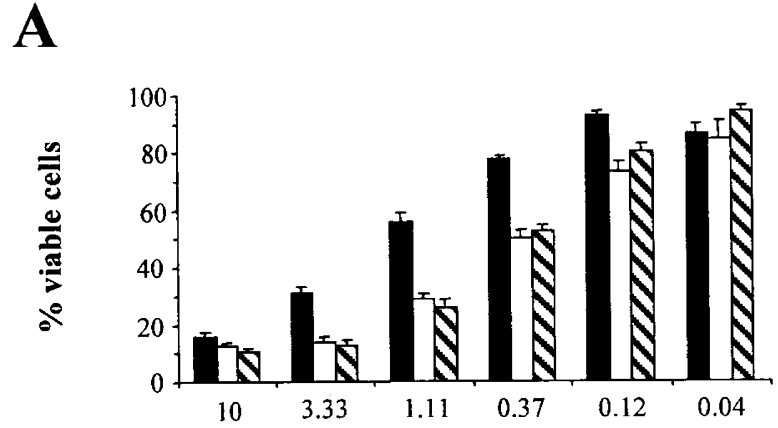

B

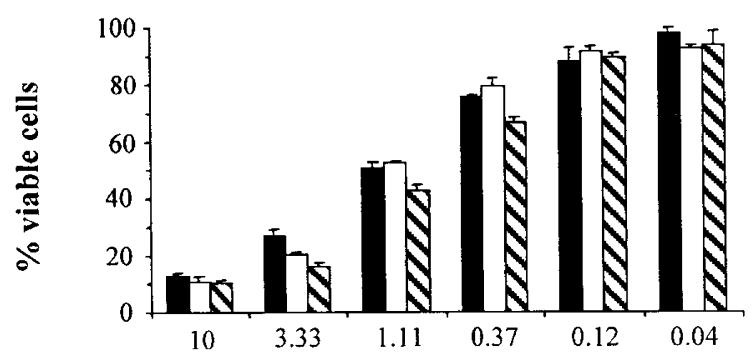

soluble FasL (ng/ml)

Figure 3 Fas-GPI enhanced Jurkat sensitivity to cytotoxicity mediated by soluble FasL and PIPLC restored basal sensitivity. Mock-transfected Jurkat cell line (filled columns) and two independent transfectants expressing a high level of Fas-GPI (open and hatched columns) were treated (A) or not (B) with PIPLC, before incubation with the indicated concentrations of soluble FasL. Viability was assessed $20 \mathrm{~h}$ later with the MTT method, and results were expressed as the percentage of cell viability in comparison to cells incubated in culture medium alone. Results are means of seven experiments \pm S.D.

in DNA fragmentation was associated to an increase in caspase activation. For this purpose, the same experiment was reproduced but aliquots of the cell mixture were lysed at $0,30,60,90$ and 120 min following the addition of the effector cells, and the production of the p10 and p14 caspase-8 activation fragments (Figure 5B, upper panel) and p17 and p20 caspase-3 activation fragments (Figure 5B, lower panel) was analyzed by immunoblotting. The activation of caspase- 8 and caspase- 3 was not detectable for mocktransfected Jurkat cells before 90 min of incubation with $1 \mathrm{~A} 12$ cells, whereas both caspases were cleaved at detectable levels as early as after $30 \mathrm{~min}$ of incubation for the cells expressing Fas-GPI. Therefore, the coengagement of Fas-GPI together with Fas accelerated Fas-mediated apoptosis, leading to a significantly increased DNA fragmentation and cell death.

\section{Fas-GPI and transmembrane Fas were localized in distinct membrane compartments on the cell surface}

It is well known that GPI-linked molecules essentially localize in membrane microdomains enriched in cholesterol and sphingolipids, the so-called lipid rafts, which are characterized by non-ionic detergent insolubility and low density. ${ }^{22,23}$ In contrast, a recent report described that the transmembrane Fas receptor is not localized in these structures. ${ }^{24}$ Therefore, we hypothesized that Fas and FasGPI could reside in distinct subdomains of the cell membrane. To verify this hypothesis, the Jurkat transfectant expressing high level of Fas-GPI was lysed with a non-ionic detergent and the membrane domains were partitioned by sucrose gradient ultracentrifugation. Nine fractions were taken from the top to the bottom of the centrifuge tube. The fractions 2 to 5 presented a visible ring containing the microdomains and the bottom fractions ( 7 to 9) accounted for the rest of the membranes. Aliquots of each fraction containing identical amounts of total protein were submitted to SDS-PAGE in denaturing conditions and revealed by Western-blotting with specific antibodies as depicted in Figure 6. Consistent with a previous study, ${ }^{25}$ the transferrin receptor (CD71) was concentrated in the membrane heavy fractions. In contrast, the p56Lck tyrosine kinase was, as previously described, ${ }^{23,26-29}$ mostly localized in the low density fractions containing the microdomains (Figure $6 \mathrm{~A}$ ). The Fas-GPI molecule could be detected using the anti-c-myc mAb, and was recovered as a $30 \mathrm{kDa}$ protein exclusively restricted to the microdomains fractions, demonstrating its association with the lipid rafts (Figure 6A). This was confirmed by immunoprecipitation with an anti-Fas mAb followed by western-blotting with a polyclonal anti-Fas antiserum (Figure 6B). In contrast, transmembrane Fas was found as a $45 \mathrm{kDa}$ protein which was exclusively restricted to the heavy membrane fractions (Figure $6 \mathrm{~B}$ ), as it has been recently reported elsewhere. ${ }^{24}$

\section{The enhancement of cell death mediated by Fas- GPI was inhibited by the protein kinase $C$ activator PMA but not by the src-kinase inhibitor PP1}

These findings raised the question of the identification of the biochemical pathway involved in this synergistic proapoptotic signal. Given the well known association between GPI-linked proteins, microdomains and src tyrosine kinases, exemplified in our system by the colocalization of p56Lck and Fas-GPI (see Figure 6), we wondered whether a src kinase specific inhibitor such as $P P 1^{30}$ could counteract the agonistic effect of Fas-GPI. PP1 at $10 \mu \mathrm{M}$ was not able to inhibit membrane FasLinduced cell death of mock-transfected Jurkat cells (Figure $7 \mathrm{~A})$, although at $1 \mu \mathrm{M}$, it abrogated the anti-CD3-triggered activation-induced cell death (results not shown) as previously found by other authors. ${ }^{30}$ Therefore, in contrast to the induction of CD3 signal transduction, the Fas pathway did not seem to be sensitive to the inhibition of the src kinase pathway. Similarly, the killing of the Fas-GPI Jurkat transfectants by the FasL effector cells was not affected by PP1 at $10 \mu \mathrm{M}$ (Figure 7B).

Phorbol esters such as PMA have been shown to inhibit apoptosis in human $\mathrm{T}$ lymphoblastoid cell lines such as Jurkat, by specifically blocking the mitochondrial step of apoptosis. ${ }^{31}$ This step can be triggered by ceramide, 

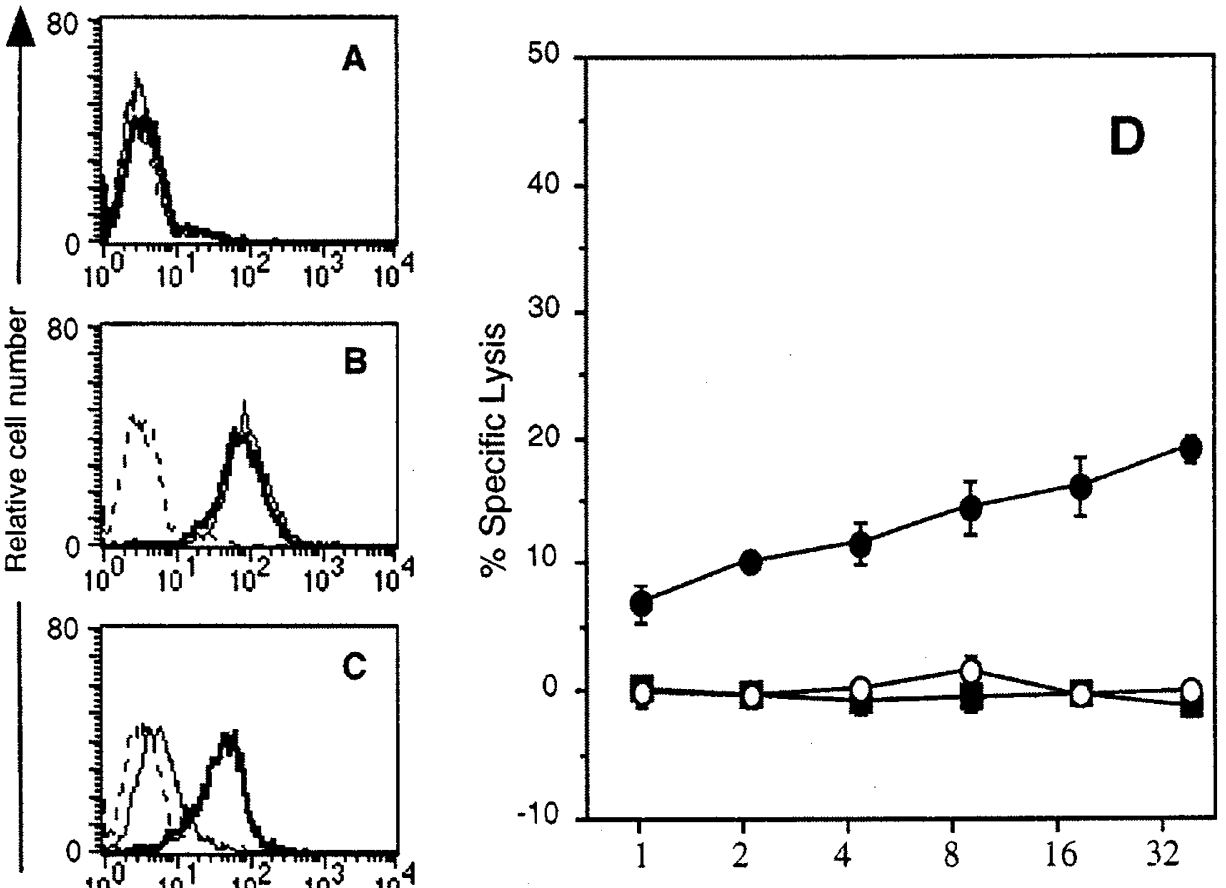

\section{Fluorescence intensity}

\section{Effector/Target ratio}

Figure 4 Fas-GPI did not trigger cell death on its own. Fas-GPI or transmembrane Fas was stably expressed in the murine T leukemic WR19L cell line, which is devoid of endogenous Fas. Fas expression was analyzed by flow cytometry on mock-transfected cells (A), one transfectant expressing Fas (B) or Fas-GPI (C), with (thin line) or without (thick line) treatment by PIPLC. Dashed line depicts the isotype-matched negative control. In (D), a ${ }^{51} \mathrm{Cr}$-release assay was performed in the presence of the $1 \mathrm{~A} 12$ effector cells, with the mock-transfected WR19L (open circles), one transfectant expressing Fas (filled circles) and one expressing Fas-GPI (filled squares). Three different transfectants expressing almost comparable levels of Fas or Fas-GPI gave similar results

A

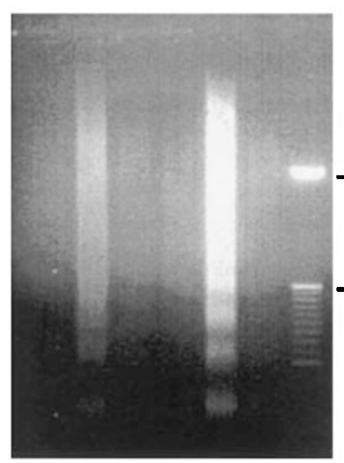

$\begin{array}{lllllll}1 & 2 & 3 & 4 & 5 & 6 & M\end{array}$
B

$\begin{array}{lllllllllll}0 & 30 & 60 & 90 & 120 & 0 & 30 & 60 & 90 & 120\end{array}$

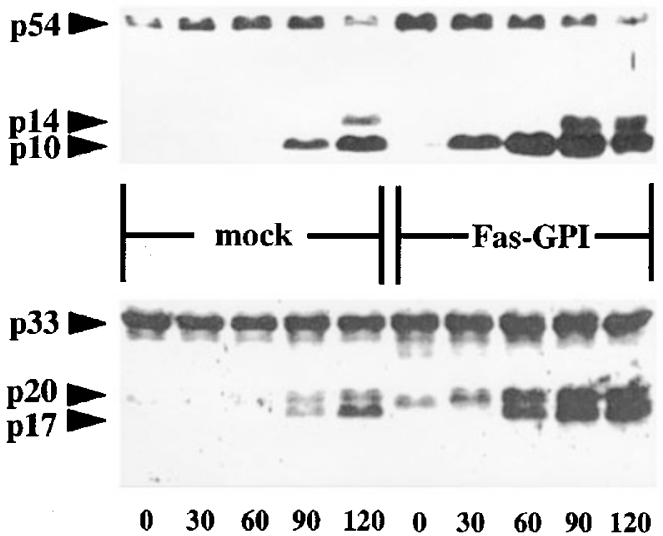

Figure 5 Fas-GPI increase of cell death was mediated by apoptosis. In (A) $3 \times 10^{6}$ mock-transfected or Fas-GPI expressing Jurkat cells were incubated with FasL-expressing (lanes 2, 3, 5 and 6) 1A12 cells or control WR19L cells (lanes 1 and 3 ) at a 1:1 ratio, for $2 \mathrm{~h}$. The incubation was performed in the presence of the anti-FasL neutralizing mAb 10F2 in lanes 3 and 6. Cell mixtures were then harvested and lysed, before DNA fragmentation was analyzed by agarose gel electrophoresis (M is a 50 bp DNA ladder marker). In (B) the cells were lysed at 0, 30, 60, 90 or 120 min after mixing and the presence of caspase 8 (upper panel) and caspase 3 (lower panel) was studied by immunoblot following separation of identical amounts of total proteins ( $45 \mu \mathrm{g}$ per lane, corresponding to around $5 \times 10^{5}$ cells). p54 is procaspase 8 and p14 and p10 are its activation products. p33 is procaspase 3 and p20 and p17 are its activation products. A non-specific band migrating between p20 and p17 is visible in the caspase 3 immunoblot 
A

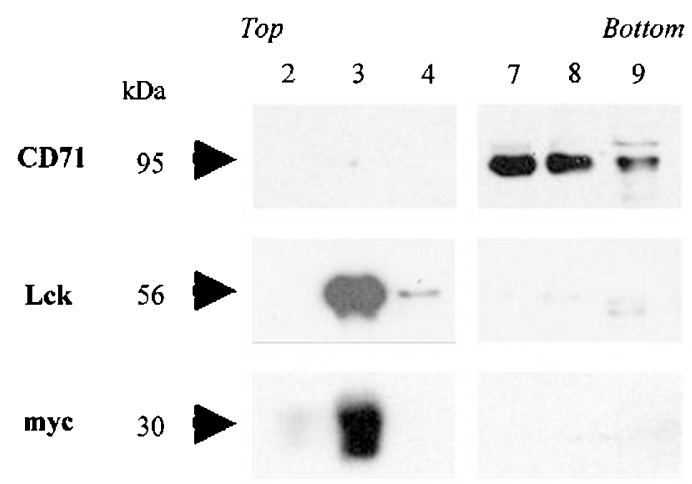

$\mathbf{B}$

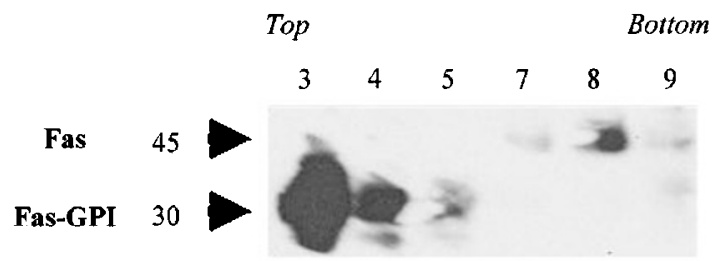

Figure 6 Fas-GPI, but not transmembrane Fas, was associated with the membrane lipid rafts. In (A) Jurkat Fas-GPI high was lysed, fractionated on a sucrose gradient as described in Material and Methods, and $10 \mu \mathrm{g}$ of total proteins for each indicated fraction were separated by SDSPAGE before immunoblotting with the indicated antibodies. In (B) $50 \mu \mathrm{g}$ of total proteins for each indicated fraction from the Jurkat Fas-GPI high were first immunoprecipitated with anti-Fas mAb (5D7), prior to SDSPAGE and immunoblotting with a goat anti-human Fas polyclonal antiserum

which originates from sphingomyelin, a sphingolipid enriched in microdomains. The mock-transfected Jurkat cell line and the Fas-GPI Jurkat transfectants were preincubated with PMA at $20 \mathrm{ng} / \mathrm{ml}$, and a ${ }^{51} \mathrm{Cr}$ release assay was carried out using the FasL expressing $1 \mathrm{~A} 12$ cell line as the effector. In these conditions, PMA had no detectable effect on the FasL-induced cell death of the mock-transfected Jurkat cells (Figure 7C). In contrast, treatment of the Jurkat Fas-GPI cells with PMA induced a substantial decrease in sensitivity to FasL, which returned to levels comparable to that obtained with the mocktransfected Jurkat cell line (Figure 7D). When Fas-GPI was cleaved off with PIPLC before the addition of PMA, the sensitivity of the Jurkat Fas-GPI transfectant to FasL became comparable to that observed for the mocktransfected cells (Figure 7D). Therefore, the Fas-GPI transfectants intrinsically lacked sensitivity to PMA, and Fas-GPI was by itself fully responsible for the acquired sensitivity to PMA. Figure 7 displays the results obtained with the Jurkat transfectant expressing a high level of FasGPI, but comparable results were found with the transfectants expressing low and intermediate levels of Fas-GPI (results not shown). Using flow cytometry, we did not detect any variation in Fas or Fas-GPI surface expression on target cells, or any fluctuation in FasL expression on the effector cells, upon treatment with PMA (results not shown). We therefore concluded from these experiments that the Fas-GPI was able to enhance Fas- mediated cell death, via the activation of a distinct signal transduction pathway specifically inhibitable by PMA, and which did not involve the microdomain associated PP1sensitive src kinases.

\section{Expression of Fas-GPI also enhanced Fas- mediated apoptosis of the CEM T-lymphoblastoid cell line}

To verify that the effect of Fas-GPI was not limited to Jurkat cells, we performed similar experiments with the human Tlymphoblastoid cell line CEM (Figure 8). Cells were stably transfected with Fas-GPI, and transfectants were isolated. In contrast to mock-transfected CEM cells, they displayed surface-bound PIPLC-sensitive Fas-GPI, although at lower amounts than that obtained with Jurkat cells (Figure 8A, for a representative transfectant). As expected, they displayed a higher sensitivity than mock-transfected CEM cells to SFasL mediated cell death (Figure 8B, histogram on the left). Treatment with PIPLC did not alter the sensitivity of mock transfected CEM cells to sFasL, while it partially inhibited cell death of the Fas-GPI expressing cells (Figure 8B, histogram on the right). Therefore, these results confirmed those obtained with the Jurkat cell line.

\section{Discussion}

We describe that an engineered GPI-linked form of Fas is capable of enhancing the cell death signal triggered via transmembrane Fas activation by membrane-bound FasL. This effect was confirmed using purified soluble FasL, and was reversed when Fas-GPI was cleaved off the cell surface by PIPLC. Although PIPLC effect appeared weaker towards sFasL than towards membrane-bound FasL, this may simply be due to the requirement for a longer incubation time with the MTT read-out. Indeed, PIPLC-cleaved Fas-GPI will be replaced by newly synthesized Fas-GPI, which might not be shed from the membrane since PIPLC is inactivated by serum components in the culture medium used for the 20-h incubation.

The agonistic effect of Fas-GPI on Fas-induced cell death could be explained by two mechanisms. Firstly, by increasing the total number of Fas binding sites for FasL on the target cell surface, the overall avidity of the FasL/Fas interaction could be augmented, leading to a more efficient delivery of the death signal via transmembrane Fas. Alternatively, Fas-GPI could actively participate to the apoptotic signalling through Fas, via the enhancement of the signal transduction pathway normally triggered by the aggregation of transmembrane Fas, may be through the activation of a distinct but synergistic pathway. This latter possibility was confirmed by the finding that PMA, a protein kinase $\mathrm{C}$ activator, was able to selectively abrogate the enhancement of cell death mediated by Fas-GPI without diminishing the response of the Jurkat control cells to the triggering of transmembrane Fas. It is striking that it has previously been reported that mutants of Fas lacking the intracellular death domain behaved as dominant negative receptors. $^{32,33}$ This is consistent with the recent observation that Fas receptors most probably exist on the cell 


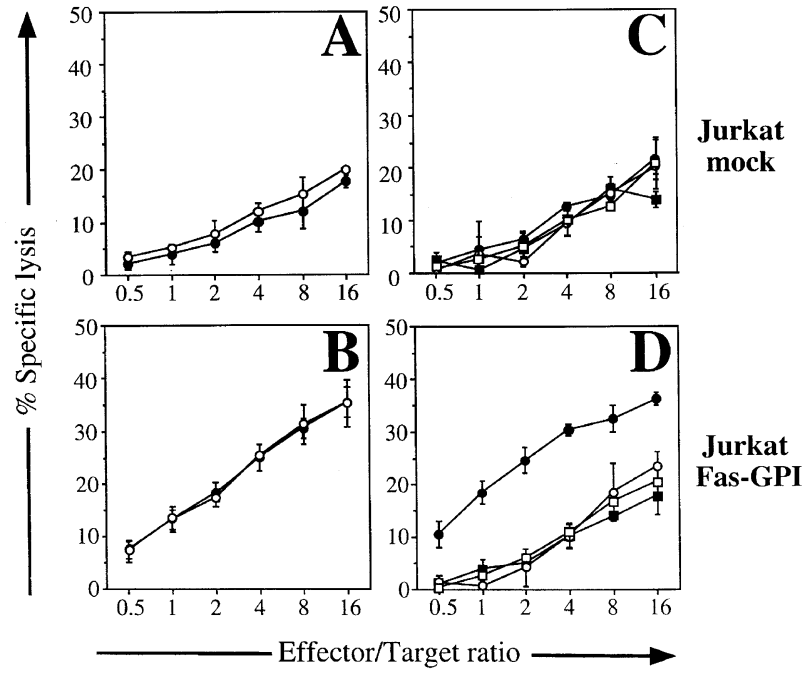

Figure 7 PMA, but not PP1, specifically inhibited the enhancement of cell death mediated by Fas-GPI. Mock-transfected (A and $\mathbf{C}$ ) or Fas-GPI high expressing (B and $\mathbf{D})$ Jurkat cells were treated (open circles) or not (closed circles) with PP1 (A and B) or PMA (C and $\mathbf{D})$. In addition, in $\mathbf{C}$ and $\mathbf{D}$, Jurkat Fas-GPI high were treated with PIPLC before addition of PMA (open squares) or not (closed squares), as described in Materials and Methods

surface as preformed homotrimers, ${ }^{34,35}$ and that the role of FasL is probably to trigger a conformational change of the trimerized Fas receptors to activate the intracellular machinery, instead of reconstituting the functional trimers as previously thought. If truncated Fas molecules lacking the intracellular region enter into the composition of heterotrimers with normal Fas receptors, therefore the triggering step mediated by FasL may be impaired, thereby explaining the dominant negative effect observed. In these cases, the truncated Fas molecules still contained the Fas transmembrane region, whereas Fas-GPI lacks it. Western blot analysis of membranes fractions demonstrated that full length Fas and Fas-GPI were localized in distinct membrane compartments, since no Fas was found in the microdomains fractions which contained Fas-GPI, and reciprocally no Fas-GPI was found in the membrane fractions containing Fas (see Figure 6). This strongly suggests that heterotrimers combining transmembrane and GPI-linked Fas do not exist on the cell surface, in contrast to what happens with death-domain deleted transmembrane Fas. In these conditions, the triggering of Fas might not be impaired, explaining why Fas-GPI did not display an inhibitory effect.

Since Fas-GPI increased Fas-mediated cell death via a PMA-sensitive pathway, we suggest that the glycolipid-rich membrane microdomains could well be involved in the signal triggered by Fas-GPI, as it has been shown in other models, such as T-lymphocyte activation. ${ }^{36-38}$ Microdomains are enriched in tyrosine kinases of the src family, which have previously been involved in Fas-mediated signal transduction by certain authors, ${ }^{39}$ but not by others. $^{40,41}$ We observed that PP1, a potent and specific inhibitor of tyrosine kinases of the src family, had no blocking effect on both Fas signal transduction and enhancement of cell death mediated by Fas-GPI, which runs against the involvement of these kinases in Fas-GPI signalling.

Although our system involving Fas-GPI is not physiological as it stands, it strongly suggests that regulatory signalling pathways of Fas-mediated apoptosis exist, which remain to be discovered. A recent report by Sieg et al. ${ }^{42}$ described that mutant effector cell lines deficient in ICAM-1 displayed a drastically decreased ability to trigger Fasinduced cell death of targets, in contrast to wild-type ICAM1 positive cells. Interestingly, LFA-1, the ICAM-1 receptor, is known to be concentrated in the microdomains. ${ }^{26}$ However, this intimate mechanism is not yet known, and may simply rely on a decrease in cell membrane adhesion, thereby impairing an efficient interaction between Fas and its ligand on the effector cell membrane. In another work, microdomains were shown to be involved in cell death triggered by TNF $-\alpha^{24}$ Indeed, the type I TNF receptor was found to be localized in the microdomains, and their disruption blocked TNF- $\alpha$ mediated apoptosis but not Fasmediated apoptosis.

The agonistic effect exerted by Fas-GPI is in striking contradiction with results obtained with the naturally existing structurally related GPI-linked TRAIL receptor DcR1, which behaves in many cellular models as a decoy receptor for TRAIL, when coexpressed together with functional TRAIL receptors. However, numerous reports did not retrieve any correlation between DcR1 expression and resistance to TRAIL. ${ }^{43-49}$ In several of these studies, intracellular factors such as the caspase 8 inhibitor cFLIP ${ }^{43,46}$ or as yet unknown factors ${ }^{48,49}$ were implicated as responsible for controlling the TRAIL receptor signal transduction pathway and therefore regulating the sensitivity to TRAIL. ${ }^{43,46,48,49}$ As a consequence, although DcR1 behaves as a decoy receptor in many cellular models, its physiological role is not yet completely known.

To our knowledge, an agonistic effect for DcR1 on TRAIL-induced cell death has never been reported. One of the many possible explanations to our findings with FasGPI could be that the membrane localization of DcR1 is different from that of Fas-GPI. DcR1 may not be localized in the microdomains, which would be unusual for a GPIlinked protein, or it may be localized in a distinct subtype of microdomains. Indeed, evidence for the coexistence of various types of microdomains, which harbor different proteins on their surface, ${ }^{26,50,51}$ has been obtained from the study of physical criteria such as size, ${ }^{26}$ relative resistance to disruption by various non-ionic detergents, ${ }^{50}$ or electron microscopy. ${ }^{51}$ Therefore, it is most conceivable that distinct subtypes of microdomains might display different functions in signal transduction.

Experiments are underway to (1) identify constitutively expressed membrane proteins localized into the microdomains, which could behave as the chimeric Fas-GPI in our experimental model, and (2) identify the pathway triggered by Fas-GPI. This would clearly demonstrate the physiological relevance of the phenomenon described here in Fasmediated apoptosis. The identification of such regulatory pathways could also help to understand why certain cell types, for example the human thymocytes, display an apparent 
A
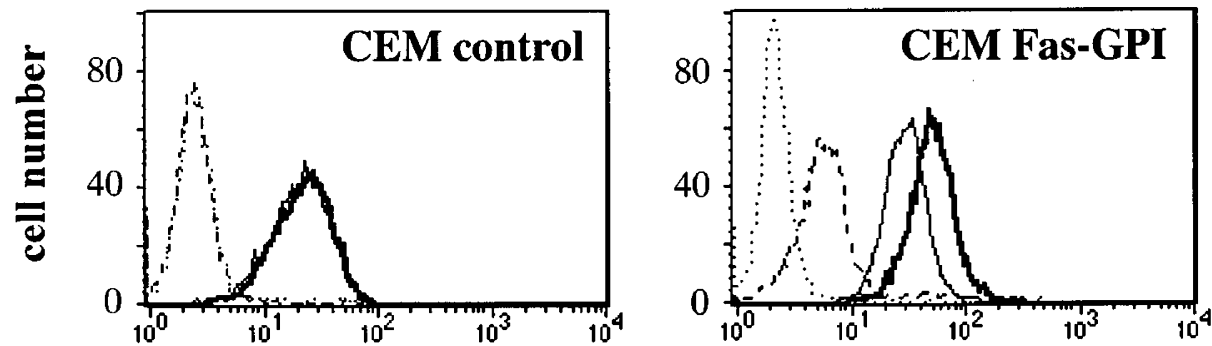

fluorescence intensity

B
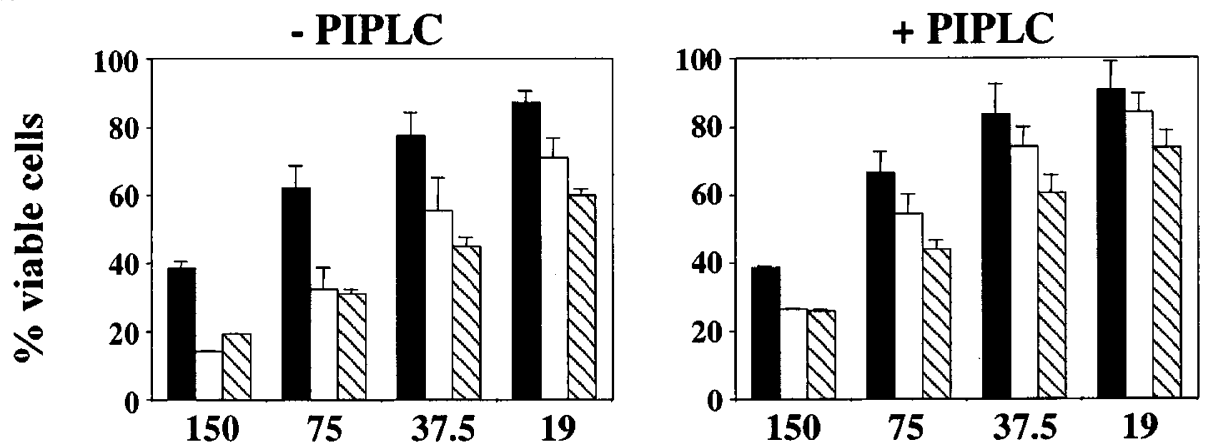

\section{soluble FasL (ng/ml)}

Figure 8 Fas-GPI also enhanced Fas-mediated cell death in CEM cells. In (A) surface staining for the c-myc epitope (dotted and dashed lines) and Fas extracellular domain (thin and thick continuous lines) were analyzed before (dashed and thick continuous lines) or after (dotted and thin continuous lines) PIPLC treatment, for the mock-transfected CEM (diagram on the left), and one representative CEM transfectant expressing high amount of Fas-GPI (diagram on the right). For the sake of clarity, isotype negative control was omitted, but was superimposed to the dotted lines in both diagrams. In (B) mock-transfected CEM (filled columns) and two independent transfectants expressing a high level of Fas-GPI (open and hatched columns) were treated (diagram on the right) or not (diagram on the left) with PIPLC, before incubation with the indicated concentrations of soluble FasL. Viability was assessed $20 \mathrm{~h}$ later with the MTT method, and results were expressed as the percentage of cell viability in comparison to cells incubated in culture medium alone. Results are means of four experiments \pm S.D.

resistance to Fas engagement despite a strong Fas expression. ${ }^{52}$ Alternatively, the engagement of such costimulation molecules, as well as the use of Fas-GPI, could represent new strategies to modulate Fas-mediated apoptosis.

\section{Materials and Methods}

\section{Cell lines and media}

The mouse T-lymphoma cell line WR19L and its transfectant $1 \mathrm{~A} 12$ expressing membrane bound $\mathrm{FasL}^{53}$ were kindly provided by $\operatorname{Pr} \mathrm{S}$ Nagata (Osaka Bioscience Institute, Osaka, Japan). The human Tlymphoblastoid cell lines CEM and Jurkat 77 were kindly provided by Dr P Anderson (Dana Farber Cancer Institute, Boston, MA, USA). All these cell lines were grown in RPMI 1640 medium supplemented with $8 \%$ heat-inactivated fetal calf serum and $2 \mathrm{mM}$ L-glutamine in a $5 \%$ $\mathrm{CO}_{2}$ incubator at $+37^{\circ} \mathrm{C}$.

\section{Antibodies and other reagents}

The fluorescein isothiocyanate (FITC)-labelled goat $F\left(a b^{\prime}\right)_{2}$ fragment anti-mouse IgG was purchased from Coulter-Immunotech (Marseille, France). Isotype control monoclonal antibody (mAb) 1F10, directed at an irrelevant antigen, was obtained in our laboratory. ${ }^{20}$ The anti-Fas $\mathrm{mAb} 5 \mathrm{D} 7$ was generated in the laboratory, following immunization of mice with a Fas-Fc fusion protein, and screening of hybridomas' supernatants with a $\mathrm{Ba} / \mathrm{F} 3$ cell line transfected with the cDNA encoding human Fas. Other anti-Fas antibodies were used, such as B-G27 and B-G30 (Diaclone, Besançon, France), DX2 (Pharmingen, San Diego, CA, USA), and a polyclonal goat anti-human Fas antiserum (R\&D Systems Europe, Oxon, UK). The anti-FasL blocking mAb 10F2 was also generated in the laboratory. The anti-c-myc mAb $9 \mathrm{E} 10$ was purified from supernatants of the hybridoma obtained from Dr Ramsay. ${ }^{54}$ Anti-transferrin mAb was from Zymed Laboratories (San Francisco, CA, USA), anti-p56Lck was from Transduction Laboratories (Lexington, KY, USA), anti-Caspase-3 was from Pharmingen and antiCaspase-8 was from R\&D Systems. Recombinant soluble human FasL (sFasL) and src kinase specific inhibitor 4-amino-5-(4- 
methylphenyl)-7-(t-butyl)pyrazolo[3,4-d]-pyrimidine (PP1) were from Alexis Corporation (Coger, Paris, France). Phosphatidylinositolspecific phospholipase C (PIPLC) and phorbol 12-myristate 13acetate (PMA) were from Sigma (L'Isle-d'Abeau-Chesnes, France).

\section{Construction of Fas-GPI and transfection of cells}

The plasmid pBS-Fas carrying the full-length human Fas antigen cDNA was a gift from Prof S Nagata. The Chimeric GPI-linked Fas receptor was constructed by subcloning the cDNA sequence encoding the extracellular region of Fas downstream to the BamHI restriction site located 14 nucleotides upstream the start of the transmembrane region, into the pEDr-gp190mycDAF plasmid ${ }^{55}$ digested to remove the gp190 coding sequence. Finally, the Fas-GPI fragment was subcloned into the pBJ1 expression vector ${ }^{19}$ which contains the $\mathrm{G} 418$ resistance gene. The Jurkat cells, the CEM cells, or the WR19L cells $\left(5 \times 10^{6}\right.$ cells in $0.8 \mathrm{ml}$ ) were transfected with $5 \mu \mathrm{g}$ of pBJ1-Fas-GPI or empty pBJ.1 vector (=mock-transfected Jurkat cells), or pBJ1-Fas (for WR19L cells only) by electroporation at $280 \mathrm{~V}$ for Jurkat and CEM or $290 \mathrm{~V}$ for WR19L with a capacitance of $900 \mu \mathrm{F}$ using an Easyject+ electroporator (Eurogentec, Seraing, Belgique). Transfected cells were resuspended in $6 \mathrm{ml}$ of growth medium. Twenty-four hours later, G418 (Life Technologies, Cergy-Pontoise, France) was added to the cultures at final concentrations of $1.9,0.5$ and $1.0 \mathrm{mg} / \mathrm{ml}$ for Jurkat, CEM and WR19L cells, respectively. After 15 days of G418 selection, the G418-resistant cell population was examined for the expression of Fas antigen and c-myc epitope and then cloned by limiting dilution to isolate transfectants expressing the recombinant Fas or Fas-GPI protein.

\section{Flow cytometry analysis of cells}

For each staining, $2 \times 10^{5}$ cells were incubated for 30 min at $+4^{\circ} \mathrm{C}$ with saturating concentrations $(10 \mu \mathrm{g} / \mathrm{ml})$ of the indicated antibody in $0.1 \mathrm{ml}$ of PBS supplemented with $1 \%$ Bovine Serum Albumin (BSA) and $0.1 \%$ human polyclonal $\operatorname{lgG}(\mathrm{w} / \mathrm{v}$, both from Sigma). The cells were then washed twice with the same buffer and incubated for $30 \mathrm{~min}$ at $+4^{\circ} \mathrm{C}$ with the FITC-conjugated goat anti-mouse IgG. After washing with PBS, the cells were resuspended in $0.14 \mathrm{ml}$ of PBS containing $1 \%$ formaldehyde $(\mathrm{v} / \mathrm{v})$ and analyzed by flow cytometry with a three color FACScalibur flow cytometer (Becton-Dickinson, Mountain View, CA, USA) equipped with the CellQuest software. For the PIPLC assays, cells $\left(1 \times 10^{6}\right)$ were treated for $1 \mathrm{~h}$ at $+37^{\circ} \mathrm{C}$ with one unit of PIPLC in serum-free medium, and were subsequently washed prior to the staining.

\section{Cell cytotoxicity assays}

The cytotoxic inducing activity of the membrane FasL-expressing (1A12) or not (WR19L) cells towards the transfected Jurkat or CEM cell lines was determined in a ${ }^{51} \mathrm{Cr}$-release assay. ${ }^{56}$ Target cells were labelled with $50 \mu \mathrm{Ci}$ of ${ }^{51} \mathrm{Cr}$ (ICN, Irvine, CA, USA) per $10^{6}$ cells, for $1 \mathrm{~h}$ at $+37^{\circ} \mathrm{C}$. Washed cells were dispensed in round-bottomed 96 well plates $\left(4 \times 10^{3}\right.$ cells per well, in triplicates) and mixed with the effector cells ( $1 \mathrm{~A} 12$ cells or WR19L parent cells) at indicated ratios, in a total volume of $0.1 \mathrm{ml}$. After $4 \mathrm{~h}$ at $37^{\circ} \mathrm{C}, 0.025 \mathrm{ml}$ of supernatants were mixed with $0.15 \mathrm{ml}$ of scintillation liquid and the released radioactivity was quantitated using a MicroBeta Trilux beta counter (Wallac, Turku, Finland). The spontaneous release of ${ }^{51} \mathrm{Cr}$ was determined by incubating the labelled target cells with medium alone, whereas the maximum release was determined by lysing the cells with $1 \%$ Triton $X$ 100 in distilled water. The per cent specific lysis was calculated as follows: [(experimental ${ }^{51} \mathrm{Cr}$ release - spontaneous ${ }^{51} \mathrm{Cr}$ release)/ (maximum ${ }^{51} \mathrm{Cr}$ release - spontaneous ${ }^{51} \mathrm{Cr}$ release)] $\times 100$. In experiments with PIPLC, the target Jurkat cells were first treated with PIPLC, then labelled with ${ }^{51} \mathrm{Cr}$, and washed to remove PIPLC before incubation with effector cells. In experiments with PP1 or PMA, the cells were first labelled with ${ }^{51} \mathrm{Cr}$, then incubated for $30 \mathrm{~min}$ with the indicated dose of the chemical, and finally mixed with the effector cells. When both PIPLC and PMA were used, the cells were treated for $1 \mathrm{~h}$ at $+37^{\circ} \mathrm{C}$ with one unit of PIPLC before the addition of PMA $(20 \mathrm{ng} / \mathrm{ml})$.

The cytotoxic activity of human SFasL was measured using the 3 [4,5-dimethylthiazol-2-yl]-2,5-diphenyltetrazolium bromide (MTT) viability assay. In brief, cells $\left(4 \times 10^{4}\right.$ per well) were cultured for $20 \mathrm{~h}$ in flat-bottomed 96 well plates with various concentrations of the apoptosis inducer. Then $0.015 \mathrm{ml}$ of MTT $(5 \mathrm{mg} / \mathrm{ml}$ in PBS) was added to each well and incubated for $4 \mathrm{~h}$ at $37^{\circ} \mathrm{C}$. The precipitates were dissolved by adding $0.115 \mathrm{ml}$ of isopropyl alcohol containing $1 \%$ formic acid (v/v), and the absorbance was measured at $570 \mathrm{~nm}$ (Titertek Labsystems Multiskan reader, Turku, Finland).

For DNA fragmentation assay and for caspase immunoblots, $3 \times 10^{6}$ target Jurkat cells were incubated for $2 \mathrm{~h}$ with $3 \times 10^{6}$ WR19L or $1 \mathrm{~A} 12$ cells. Cells were lysed in $1 \%$ Triton X-100 lysis buffer containing protease inhibitors, and the insoluble pellet was discarded after centrifugation. For caspase immunoblots, the supernatant was analysed after protein quantitation. For DNA fragmentation assay, the supernatant was precipitated with 2 volumes of isopropanol, the pellet was washed, dried and resuspended in TE buffer in the presence of RNase, before analysis on a $1.5 \%$ agarose gel, as described previously. ${ }^{57}$

\section{Isolation of the membrane microdomains}

Membrane microdomains were isolated according to the method of Ko et al. ${ }^{24}$ Briefly, $5 \times 10^{8}$ cells were rapidly chilled in liquid nitrogen and washed with Phosphate-Buffered Saline (PBS). The cells were incubated with $1 \mathrm{ml}$ of HEPES buffer (25 mM HEPES, $150 \mathrm{mM}$ $\mathrm{NaCl}, \mathrm{pH} 7.4)$ containing $1 \%$ Triton $\mathrm{X}-100$ and protease inhibitors (1 $\mathrm{mM}$ PMSF, $5 \mu \mathrm{g} / \mathrm{ml}$ Aprotinin, $10 \mu \mathrm{M}$ Leupeptin) for $30 \mathrm{~min}$ at $4{ }^{\circ} \mathrm{C}$. The lysate was mixed with an equal volume of $85 \%$ sucrose $(w / v)$ in HEPES buffer, transferred to a centrifuge tube and overlaid with $5 \mathrm{ml}$ of a $30 \%$ sucrose and $3 \mathrm{ml}$ of a $5 \%$ sucrose solutions. The sucrose gradient was centrifuged for $21 \mathrm{~h}+4^{\circ} \mathrm{C}$ in a Kontron TST4114 rotor at $250000 \mathrm{~g}_{\max }$, and nine $1 \mathrm{ml}$ fractions were harvested separately.

\section{Immunoblotting experiments}

For membrane fractions and total cell lysates, respectively 10 or $45 \mu \mathrm{g}$ of protein were separated by SDS -PAGE in reducing conditions on 12 or $13 \%$ gels, and transferred to a PVDF membrane (Amersham Pharmacia Biotech, Buckinghamshire, UK). The membrane was blocked $1 \mathrm{~h}$ with TBST (50 mM Tris, $160 \mathrm{mM} \mathrm{NaCl}, 0.1 \%$ Tween 20, $\mathrm{pH} 8$ ) containing $5 \%$ dried skimmed milk, and all subsequent steps were performed in this buffer. The indicated specific antibody was then incubated overnight at $4^{\circ} \mathrm{C}$. After four washes, the peroxydaselabelled anti-mouse (Amersham), anti-goat (Vector Laboratories, Burlingame, CA, USA) or anti-rabbit (Zymed) secondary antibody was added for $1 \mathrm{~h}$. Then the proteins were visualized with the enhanced chemiluminescence substrate kit (ECL, Amersham). For the immunoprecipitation experiments, $50 \mu \mathrm{g}$ of proteins from the indicated fractions were first immunoprecipitated with $10 \mu \mathrm{g}$ of anti-Fas 5D7 $\mathrm{mAb}$ in the presence of protein G-Sepharose beads (Sigma) overnight at $4{ }^{\circ} \mathrm{C}$. The beads were washed four times in HEPES buffer containing $0.5 \%$ Triton X-100 (Sigma). The immunoprecipitated proteins were analyzed by SDS-PAGE as described above. 


\section{Acknowledgements}

We are indebted to Pr S Nagata who kindly provided us with the WR19L and $1 \mathrm{~A} 12$ cell lines, and with the cDNA encoding human Fas. We thank Dr P Anderson who kindly provided us with the Jurkat cell line. This work was supported by grants from the Comité des Charentes de la Ligue Nationale contre le Cancer, the Comité de la Gironde de la Ligue Nationale contre le Cancer and the Association pour la Recherche contre le Cancer.

\section{References}

1. Vignaux F and Golstein P (1994) Fas-based lymphocyte-mediated cytotoxicity against syngeneic activated lymphocytes: a regulatory pathway? Eur. J. Immunol. 24: 923-927

2. Alderson MR, Tough TW, Davis-Smith T, Braddy S, Falk B, Schooley KA, Goodwin RG, Smith CA, Ramsdell F and Lynch DH (1995) Fas ligand mediates activation-induced cell death in human T lymphocytes. J. Exp. Med. 181:71-77

3. Dhein J, Walczak H, Bäumler C, Debatin KM and Krammer PH (1995) Autocrine T-cell suicide mediated by Apo-1 (Fas/CD95). Nature 373: 438-441

4. Koppi TA, Tough-Bement T, Lewinsohn DM, Lynch DH and Alderson MR (1997) CD40 ligand inhibits Fas/CD95-mediated apoptosis of human blood-derived dendritic cells. Eur. J. Immunol. 27: 3161-3165

5. Matsue H, Edelbaum D, Hartmann AC, Morita A, Bergstresser PR, Yagita $H$ Okumura K and Takashima A (1999) Dendritic cells undergo rapid apoptosis in vitro during antigen-specific interaction with CD4+T cells. J. Immunol. 162: $5287-5298$

6. Kawamura T, Azuma M, Kayagaki N, Shimada S, Yagita Hand Okumura K(1999) Fas/Fas ligand-mediated elimination of antigen-bearing Langerhans cells in draining lymph nodes. Br. J. Dermatol. 141: 201-205

7. Ashkenazi A and Dixit VM (1999) Apoptosis control by death and decoy receptors. Current Opin Cell Biol. 11: 255-260

8. Degli-Esposito MA, Smolak PJ, Walczak H, Waugh J, Huang CP, DuBose RF, Goodwin RG and Smith CA (1997) Cloning and characterization of TRAIL-R3, a novel member of the emerging TRAIL receptor family. J. Exp. Med. 186: 11651170

9. McFarlane M, Ahmad M, Srinivasula SM, Fernandes-Alnemri T, Cohen GM and Alnemri ES (1997) Identification and molecular cloning of two novel receptors for the cytotoxic ligand TRAIL. J. Biol. Chem. 272: 25417-25420

10. Pan G, Ni J, Wei YF, Yu GL, Gentz R and Dixit VM (1997) An antagonist decoy receptor and a death domain-containing receptor for TRAIL. Science 277: 815818

11. Mongkolsapaya J, Cowper AE, Xu XN, Morris G, McMichael AJ, Bell Jl and Screaton GR (1998) Lymphocyte inhibitor of TRAIL (TNF-related apoptosisinducing ligand): a new receptor protecting lymphocytes from the death ligand TRAIL. J. Immunol. 160: 3-6

12. Sheridan JP, Marsters SA, Pitti RM, Gurney A, Skubatch M, Baldwyn D, Ramakrishnan L, Gray CL, Baker K, Wood WI, Goddard AD, Godowski P and Ashkenazi A (1997) Control of TRAIL-induced apoptosis by a family of signaling and decoy receptors. Science 277: 818-821

13. Schneider P, Bodmer JL, Thome M, Hofmann K, Holler N and Tschopp J (1997) Characterization of two receptors for TRAIL. FEBS Lett. 416: 329-334

14. Truneh A, Sharma S, Silverman C, Khandekar S, Reddy MP, Deen KC McLaughlin MM, Srinivasula SM, Livi GP, Marshall LA, Alnemri ES, Williams WV and Doyle ML (2000) Temperature-sensitive differential affinity of TRAIL for its receptors. DR5 is the highest affinity receptor. J. Biol. Chem. 275: 23319-23325

15. Zhang XD, Franco AV, Nguyen T, Gray CP and Hersey P (2000) Differential localization and regulation of death and decoy receptors for TNF-related apoptosis-inducing ligand (TRAIL) in human melanoma cells. J. Immunol. 164 $3961-3970$

16. Bernard D, Quatannens B, Vandenbunder B and Abbadie C (2001) Rel/NFkappaB transcription factors protect against tumor necrosis factor (TNF)-related apoptosis-inducing ligand (TRAIL)-induced apoptosis by up-regulating the TRAIL decoy receptor DcR1. J. Biol. Chem. 276: 27322-27328

17. Moran P and Caras IW (1991) Fusion of sequence elements from non-anchored proteins to generate a fully functional signal for glycophosphatidylinositol membrane anchor attachment. J. Cell. Biol. 115: 1595-1600
18. Caras IW (1991) An internally positioned signal can direct attachment of a glycophospholipid membrane anchor. J. Cell. Biol. 113: 77-85

19. Lin A, Devaux B, Green A, Sagerström C, Elliott JF and Davis MM (1990) Expression of $T$ cell antigen receptor heterodimers in a lipid-linked form. Science 249: $677-679$

20. Taupin JL, Acres B, DottK, SchmittD, Kieny MP, Gualde Nand Moreau JF (1993) Immunogenicity of HILDA/LIF either in a soluble or in a membrane anchored form expressed in vivo by recombinant vaccinia viruses. Scand. J. Immunol. 38: 293301

21. Hannan LA and Editin M (1996) Traffic, polarity, and detergent solubility of a glycosylphosphatidylinositol-anchored protein after LDL-deprivation of MDCK cells. J. Cell. Biol. 133: 1265-1276

22. Simons K and lkonen E (1997) Functional rafts in cell membranes. Nature 387: 569-572

23. Friedrichson T and Kurzchalia TV (1998) Microdomains of GPI-anchored proteins in living cells revealed by crosslinking. Nature 394: 802-805

24. Ko YG, Lee JS, Kang YS, Ahn JH and Seo JS (1999) TNF- $\alpha$-mediated apoptosis is initiated in caveolae-like domains. J. Immunol. 162: 7217-7223

25. Mane S, Mira E, Gomez-Mouton C, Lacalle RA, Keller P, Labrador JP and Martinez AC (1999) Membrane raft microdomains mediate front-rear polarity in migrating cells. EMBO J. 18: 6211-6220

26. Cerny J, Stockinger $H$ and Horejsi V (1996) Noncovalent associations of $T$ lymphocyte surface proteins. Eur. J. Immunol. 26: 2335-2343

27. Harder T and Simons K (1997) Caveolae, DIGs, and the dynamics of sphingolipid-cholesterol microdomains. Curr. Opin. Cell. Biol. 9: 534-542

28. Varma R and Mayor S (1998) GPI-anchored proteins are organized in submicron domains at the cell surface. Nature 394: 798-801

29. Brown DA and London E (1998) Functions of lipid rafts in biological membranes. Annu. Rev. Cell. Dev. Biol. 14: 111-136

30. Hanke JH, GardnerJP, L. DR, Changelian PS, BrissetteWH, WeringerEJ, Pollok BA and Connelly PA (1996) Discovery of a novel, potent, and Src family-selective tyrosine kinase inhibitor. Study of Lck- and FynT-dependent T cell activation. J. Biol. Chem. 271: 695-701

31. Scaffidi C, Schmitz I, Zha J, Korsmeyer SJ, Krammer PH and Peter ME (1999) Differential modulation of apoptosis sensitivity in CD95 type I and type Il cells. J. Biol. Chem. 274: 22532-22538

32. Cascino I, Papoff G, De Maria R, Testi R and Ruberti G (1996) Fas/Apo-1 (CD95) receptor lacking the intracytoplasmic signalling domain protects tumor cells from Fas-mediated apoptosis. J. Immunol. 156: 13-17

33. Rieux-Laucat F, Le Deist F, Hivroz C, Roberts IA, Debatin KM, Fischer A and de Villartay JP (1995) Mutations in Fas associated with human lymphoproliferative syndrome and autoimmunity. Science 268: $1347-1349$

34. Papoff G, Hausler P, Eramo A, Pagano MG, Di Leve G, Signore A and Ruberti G (1999) Identification and characterization of a ligand-independent oligomerization domain in the extracellular region of the CD95 death receptor. J. Biol. Chem. 274: $38241-38250$

35. Siegel RM, Frederiksen JK, Zacharias DA, Chan FK, Johnson M, Lynch D, Tsien RY and Lenardo MJ (2000) Fas preassociation required for apoptosis signaling and dominant inhibition by pathogenic mutations. Science 288: 2354-2357

36. Viola A, Schroeder S, Sakakibara Y and Lanzavecchia A (1999) T lymphocyte costimulation mediated by reorganization of membrane microdomains. Science 283: $680-682$

37. Montixi C, Langlet C, Bernard AM, Thimonier J, Dubois C, Wurbel MA, Chauvin JP, Pierres M and He HT (1998) Engagement of T cell receptor triggers its recruitment to low-density detergent-insoluble membrane domains. EMBOJ.17: $5334-5348$

38. Xavier R, Brennan T, Li Q, McCormack C and Seed B (1998) Membrane compartmentation is required for efficient T cell activation. Immunity 8: 723-732

39. Schlottmann KE, Gulbins E, Lau SM and Coggeshall KM (1996) Activation of Srcfamily tyrosine kinases during Fas-induced apoptosis. J. Leukoc. Biol. 60:546554

40. Oyaizu N, Than S, McCloskey TW and Pahwa S(1995) Requirement of p561ck in T-cell receptor/CD3-mediated apoptosis and Fas- ligand induction in Jurkat cells. Biochem. Biophys. Res. Commun. 213: 994-1001

41. Latinis KM and Koretzky GA (1996) Fas ligation induces apoptosis and Jun kinase activation independently of CD45 and Lck in human T cells. Blood 87: $871-875$

42. Sieg S, Smith D and Kaplan D (1999) Differential activity of soluble versus cellular Fas ligand: regulation by an accessory molecule. Cell. Immunol. 195: 89-95 
43. Griffith TS, Chin WA, Jackson GC, Lynch DH and Kubin MZ (1998) Intracellular regulation of TRAIL-induced apoptosis in human melanoma cells. J. Immunol. 161: 2833-2840

44. Griffith TS, Rauch CT, Smolak PJ, Waugh JY, Boiani N, Lynch DH, Smith CA, Goodwin RG and Kubin MZ (1999) Functional analysis of TRAIL receptors using monoclonal antibodies. J. Immunol. 162: 2597-2605

45. Mitsiades N, Poulaki V, Mitsiades C and Tsokos M (2001) Ewing's sarcoma family tumors are sensitive to tumor necrosis factor-related apoptosis-inducing ligand and express death receptor 4 and death receptor 5. Cancer Res. 61: 2704-2712

46. Leverkus M, Neumann M, Mengling T, Rauch CT, Brocker EB, Krammer PH and Walczak H (2000) Regulation of tumor necrosis factor-related apoptosisinducing ligand sensitivity in primary and transformed human keratinocytes. Cancer Res. 60: 553-559

47. Wendling U, Walczak H, Dorr J, Jaboci C, Weller M, Krammer PH and Zipp F (2000) Expression of TRAIL receptors in human autoreactive and foreign antigen- specific T cells. Cell Death Differ. 7: 637-644

48. Zhang XD, Nguyen T, Thomas WD, Sanders JE and Hersey $P(2000)$ Mechanisms of resistance of normal cells to TRAIL induced apoptosis vary between different cell types. FEBS Lett. 482: 193-199

49. Zhang XD, Franco A, Myers K, Gray C, Nguyen T and Hersey P (1999) Relation of TNF-related apoptosis-inducing ligand (TRAIL) receptor and FLICE-inhibitory protein expression to TRAIL-induced apoptosis of melanoma. Cancer Res. 59: $2747-2753$
50. Röper K, Corbeil D and Huttner WB (2000) Retention of prominin in microvilli reveals distinct cholesterol-based lipid microdomains in the apical plasma membrane. Nature Cell. Biol. 2: 582-592

51. Madore N, Smith KL, Graham CH, Jen A, Brady K, Hall S and Morris R (1999) Functionally different GPI proteins are organized in different domains on the neuronal surface. EMBO J. 18: 6917-6926

52. Jenkins M, Keir M and McCune JM (1999) Fas is expressed early in human thymocyte development but does not transmit an apoptotic signal. J. Immunol. 163: $1195-1204$

53. Tanaka M, Suda T, Haze K, Nakamura N, Sato K, Kimura F, Motoyoshi K, Mizuki M, Tagawa S, Ohga S, Hatake K, Drummond AH and Nagata S (1996) Fas ligand in human serum. Nat. Med. 2: 317-322

54. Evan GI, Lewis GK, Ramsay G and Bishop JM (1985) Isolation of monoclona antibodies specific for human c-myc proto-oncogene product. Mol. Cell. Biol. 5 3610-3616

55. Pitard V, Taupin JL, Miossec V, Blanchard F, Cransac M, Jollet I, Vernallis A Hudson K, Godard A, Jacques $Y$ and Moreau JF (1997) Production and characterization of monoclonal antibodies against the leukemia inhibitory factor low affinity receptor, gp 190. J. Immunol. Meth. 205: 177-190

56. Suda T and Nagata S (1994) Purification and characterization of the Fas-ligand that induces apoptosis. J. Exp. Med. 179: 873-879

57. Tian Q, Taupin J, Elledge S, Robertson M and Anderson P (1995) Fas-activated serine/threonine kinase (FAST) phosphorylates TIA-1 during Fas-mediated apoptosis. J. Exp. Med. 182: 865-874 